\title{
Lessons learned. Teil 1
}

\author{
Die Schaffung eines öffentlichen Spitalverbundes in der Form einer Aktien- \\ gesellschaft am Beispiel der Spital Thurgau AG aus Sicht des Präsidenten der \\ Thurgauischen Vereinigung Leitender Spitalärzte.
}

\section{R. O. Binswanger}

Präsident der Thurgauischen Vereinigung Leitender Spitalärzte

\author{
Vorbereitung, Gründung, \\ Entwicklung der Spital Thurgau AG \\ in den ersten Jahren
}

\section{Ausgangslage}

Im Jahre 1998 versammelte der damalige Vorsteher des Departements für Finanzen und Soziales (DFS) des Kantons Thurgau die Kaderärzte des Kantonsspitals Frauenfeld (KSF), des Kantonsspitals Münsterlingen (KSM), der Psychiatrischen Dienste Thurgau Münsterlingen (PDT) sowie der Rehabilitationsklinik St. Katharinental Diessenhofen (TKK), um ihnen zu eröffnen, dass er beabsichtige, aus den Spitälern die privatrechtliche Spital Thurgau AG (STGAG) zu bilden. Das vorgängig erarbeitete Konzept wurde nach intensiver Debatte vom Kantonsrat genehmigt.

Die STGAG wurde geschaffen, der Verwaltungsrat und die Geschäftsleitung gewählt. Die Aktiengesellschaft übernahm die Spitäler per 1. Januar 2000.

\section{Ziele}

In die Umwandlung wurden u.a. folgende Erwartungen gesetzt:

- Elimination der Mehrfachrollen des Kantons als Spitalplaner, Leistungseinkäufer und Leistungserbringer;

- Entflechtung der Verantwortlichkeiten zwischen dem DFS und den Spitälern;

- Erhöhung der Flexibilität und Schlagkraft des Spitalverbundes;

- Beschleunigung der Entscheidungsprozesse;

- Verbesserung der Infrastruktur, speziell des Rechnungswesens und der Informationstechnologie;

- Aufzeigen und die Realisierung von Sparpotentialen.

\section{Aktivitäten und Postulate der TVLS}

Die Thurgauische Vereinigung Leitender Spitalärzte (TVLS) bestand zum Zeitpunkt der Gründung der Aktiengesellschaft schon mehrere Jahre. Sie entfaltete eine rege Tätigkeit und formulierte folgende Postulate:

- Anerkennung der TVLS als eigenständiger Arbeitnehmerverband innerhalb der STGAG;
- Forderung nach mindestens einem aussenstehenden Arzt als Mitglied des Verwaltungsrates;

- angemessene Vertretung der Ärzteschaft in der Geschäftsleitung;

- Entlastung der Ärzte von administrativen Arbeiten.

\section{Strukturen}

Der Kanton ist zu 100\% Eigentümer der STGAG. Er wird vertreten durch den Vorsteher des DFS. Der Verwaltungsrat bestand zunächst aus 5 Mitgliedern (dem Geschäftsleiter einer schaffhausischen Firma mit Aktivitäten im Gesundheitswesen, einem Vertreter einer Beratungsfirma im Gesundheitswesen, dem ehemaligen Vorsteher des DFS und Ständerat, der Direktorin eines Universitätsspitals sowie einem Anwalt, der damals Sekretär der Thurgauischen Ärztegesellschaft war).

Die Geschäftsleitung bestand zunächst aus sechs Mitgliedern, nämlich dem Geschäftsleiter (CEO), dem Finanzchef, den Spitaldirektoren (gleichzeitig Verwaltungsdirektoren) des KSF und KSM sowie den ärztlichen Direktoren (Spitaldirektoren in Personalunion) der PDT und der TKK.

Das Gesundheitsamt des Kantons Thurgau formulierte den Rahmenkontrakt zwischen dem Kanton und der STGAG. Er kontrolliert auch dessen Einhaltung. Ein Beirat sorgt für eine breitere politische Abstützung (Abb. 1 und 2).

\section{Strategiefragen}

Verwaltungsrat und Geschäftsleitung entfalteten rasch eine intensive Strategiediskussion, für welche die obengenannte Beratungsfirma wesentliche Inputs lieferte und die teilweise von Mitgliedern der Beratungsfirma begleitet wurde. Hauptfokus der strategischen Vorgaben war das Schaffen und Ausnützen von Synergien, die sich hauptsächlich auf die beiden Kantonsspitäler bezogen. Dabei wurde wenigstens teilweise ein Modell favorisiert, das eine Zusammenfassung von Kliniken der beiden Spitäler unter einem einzigen Chefarzt vorsah, wobei vor allem die Spezialkliniken, u.a. die Urologie, Orthopädie und die Radiologie, in Betracht gezogen wurden. In paradigmatischer Weise wurde das Prinzip der 
Einerführung auf allen Hierarchieebenen vorgeschrieben. Die TVLS stellte zu Strategiefragen eigene Überlegungen an, und der Präsident legte dazu ein Muster mit Varianten vor (Tab. 1).

\section{Kaderarztvertrag}

Sehr bald nach der Gründung der STGAG wurden Verhandlungen zu einem neuen Kaderarztvertrag aufgenommen. Dieser sollte die Anstellungsbedingungen der Chefärzte, der Leitenden Ärzte sowie der Beleg- und Konsiliarärzte regeln, und zwar in Anlehnung an den gleichzeitig in Auftrag gegebenen neuen Gesamtarbeitsvertrag

Abbildung 1: Organigramm alt

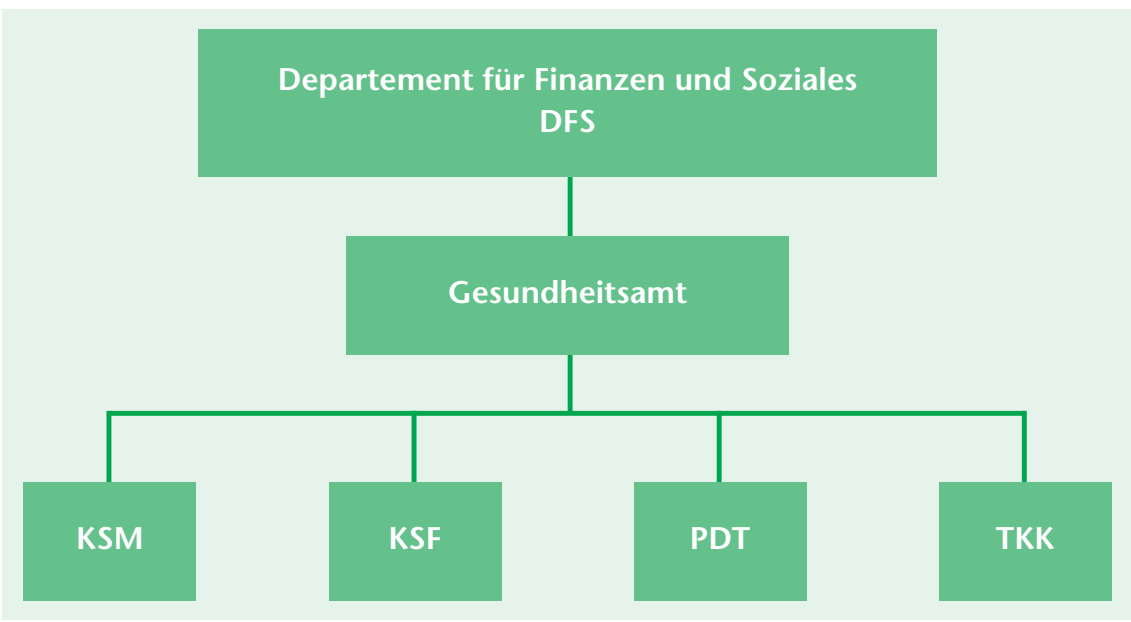

Abbildung 2: Organigramm neu

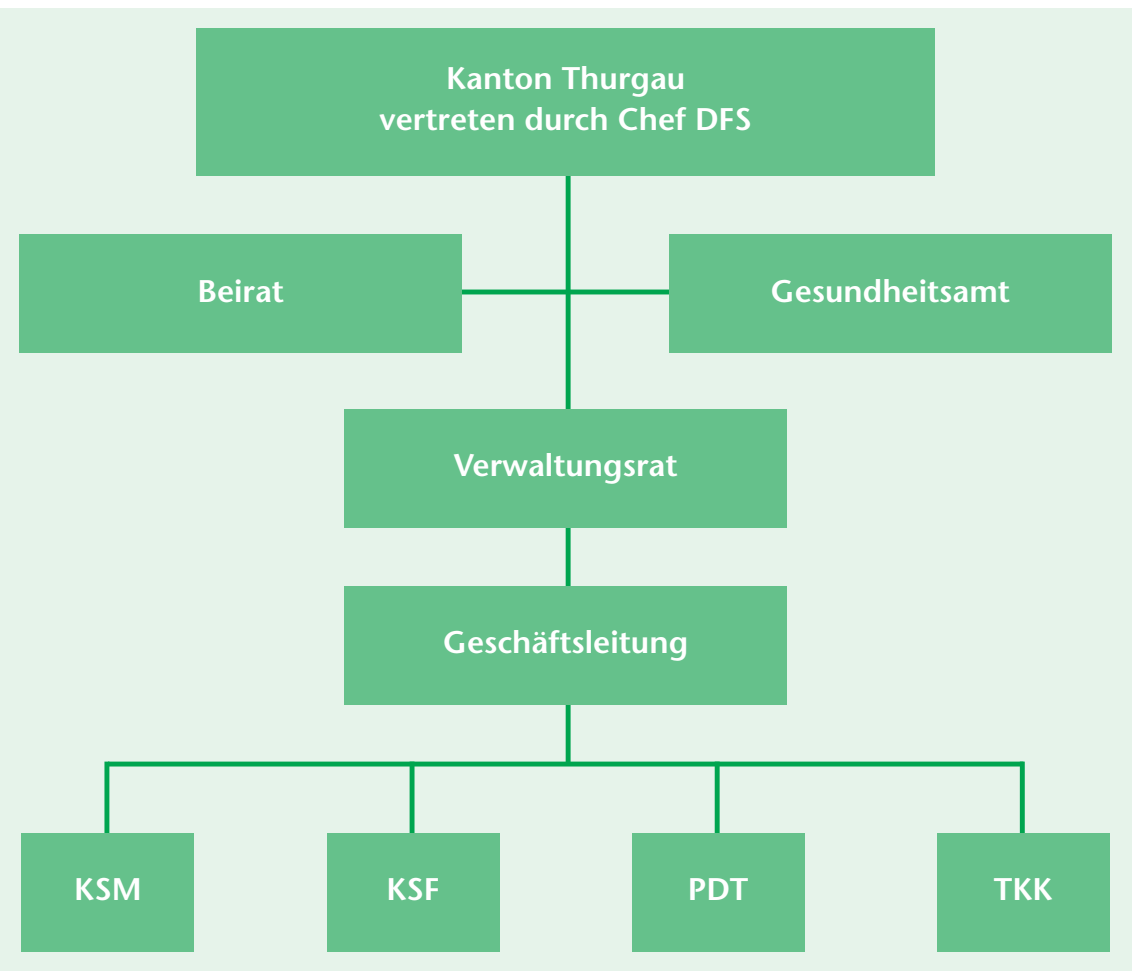

für die übrigen Mitarbeiter (Firmenvertrag), mit speziellen Modalitäten für die Kaderärzte, insbesondere dem Recht der Privatliquidation. Ausdrückliches Postulat der STGAG war das Einbringen einer erfolgsbezogenen Abgeltung der Kaderärzte. In bezug auf die Gesamtabgeltung der Kaderärzte wurde eine Kosten- bzw. Ertragsneutralität vereinbart.

Infolge der komplexen damit verbundenen Fragestellungen wurde während ca. eines Jahres intensiv verhandelt. Ein Grundsatzpapier wurde erstellt und verabschiedet. Es stellte sich heraus, dass so viele Abhängigkeiten vom neuen ambulanten Tarif TARMED bestanden, dass dessen Auswirkungen zunächst abgewartet werden sollten. In einer 2. Runde wurde im Jahr 2004 der Kaderarztvertrag finalisiert und trat per 1. Januar 2005 in Kraft.

\section{Externe Beratung}

Der Zuzug externer Berater für Strategie und Projekte war ausdrücklich vorgesehen und spielte im folgenden eine grosse Rolle. Im Anschluss an eine Tarifsenkung, welche die Ertragskraft der STGAG erheblich tangierte, erhielt die Beratungsfirma einen grossen Beratungsauftrag im Jahre 2003. Dieses Projekt sollte in möglichst kurzer Zeit Einsparungen aufzeigen und realisieren. Im Rahmen des Projektes wurden 100 Mitarbeiter der Spitäler und Kliniken befragt, darunter eine grosse Zahl von Kaderärzten. Als Resultat der Projektarbeit wurde eine Auswertung vorgelegt, die mögliche Einsparungen im Betrag von 20 Millionen Franken pro Jahr aufzeigte bei einem Gesamtumsatz von etwa 350 Millionen Franken. Einzelne Massnahmen wurden realisiert und haben zu Einsparungen geführt, allerdings in geringerem Ausmass als prognostiziert.

\section{Projekte}

Geschäftsleitung und Verwaltungsrat definierten innerhalb etwa eines Jahres 22 Projekte, die nach einem vorgegebenen Projektablauf in Angriff genommen wurden. Von diesen Projekten sind bis heute mehrere realisiert worden.

\section{Vorschläge der TVLS}

Mit der Überführung der kantonalen Spitäler und Kliniken in eine Aktiengesellschaft betrat der Kanton Thurgau Neuland. Entsprechend gross war der Bedarf an Anpassungen in den ersten Jahren. Die Mitglieder des TVLS leisteten dazu einen grossen Beitrag und legten Anfang 2004 dem Departementsvorsteher des DFS und dem Verwaltungsrat einen detaillierten Katalog von Massnahmen vor, die zum Ziel hatten, die Institution und damit ihre Schlagkraft zu verbessern. Wesent- 
liche Teile dieser Vorschläge wurden auch von den Organen der STGAG im Vorfeld erkannt, so dass eine beträchtliche Anzahl der Postulate im Jahre 2004 und 2005 realisiert wurde.

\section{Personelle Veränderungen}

Im Jahre 2004 schieden der Verwaltungsratspräsident und der CEO aus geschäftlichen bzw. privaten Gründen aus, was zum Anlass genommen wurde, gewisse Struktur- und Personalveränderungen durchzuführen. So wurden ein Finanzfachmann und ein ausserkantonaler Arzt in den Verwaltungsrat aufgenommen. Zusätzlich traten die ärztlichen Direktoren des KSF und KSM in die Geschäftleitung ein. Somit konnten die Postulate der ärztlichen Vertretung im Verwaltungsrat und der Repräsentanz der Ärzteschaft in der Geschäftsleitung realisiert bzw. verstärkt werden.

\section{Infrastruktur}

Grosse Anstrengungen wurden unternommen, um die Infrastruktur, speziell im Bereich der Informationstechnologie und des Rechnungswesens, zu verbessern, was im ersteren Falle weitgehend gelungen ist, im zweiten Falle kurz vor der Realisierung steht.

Eine weitere Entwicklung geht in Richtung einer spitalübergreifenden administrativen Führung, die teilweise realisiert ist.

\section{Kommunikation}

Aufgrund der komplexeren Strukturen mit Einführen zweier neuer Führungsebenen ergab sich ein hoher Kommunikations- und Informationsbedarf.

Schaltstelle der Kommunikation war die Geschäftsleitung, insbesondere der $\mathrm{CEO}$, bei dem alle Informationen aus Verwaltungsrat bzw. den nachgeordneten Führungsebenen zusammenliefen und nach oben bzw. nach unten weitergegeben wurden. Dabei wurde zunächst auf einen genauen Formalismus geachtet u.a. mit dem Ziel, die Informationen und die Kommunikation strikt auf dem Dienstweg und nicht ausserhalb desselben zu halten.

\section{Unternehmungskulturelle Aspekte}

Für die neu eintretenden Organe der Gesellschaft ergab sich ein sehr grosser Informations- und Analysebedarf, dem auf seiten der Kaderärzte ein grosser Erfahrungsschatz gegenüberstand. In den ersten Jahren überwog diese Analysekultur ganz klar die bestehende Erfahrungskultur. Es kam zu unterschiedlichen Sichtweisen in den Fragen der Strategie und Strukturen zwischen den «Neueintretenden» und den «Alteingesessenen». Daraus entwickelte sich im Endeffekt eine positive Auseinandersetzung, die inzwischen ihr Gleichgewicht gefunden hat in einer Mischung von Analyse- und Erfahrungskultur. Die AG-Lösung hat zu einer Entpolitisierung geführt, indem das Parlament praktisch keinen Einfluss mehr auf die Spitäler nimmt. Das DSF steht aber nach wie vor noch in der Verantwortung für die Höhe der Gesundheitsausgaben.

\section{Rolle des DFS und Gesundheitsamtes}

Dem DFS fällt die Rolle des Eigentümers zu. Es ist aufgefordert, eine Eigentümerstrategie zu entwickeln. Dabei stellte es sich auf den Standpunkt, dass die Eigentümerstrategie mit den Leistungsaufträgen ausreichend vorgegeben ist. Für die Kaderärzte greift diese Sichtweise zu kurz. Die Kaderärzte haben deshalb vier mögliche Strategien formuliert (Tab. 1). Ohne explizit dazu Stellung zu nehmen, wird aktuell die Retailstrategie verfolgt.

Tabelle 1

Strategiekonzept STGAG aus Sicht des Präsidenten TVLS.

\begin{tabular}{|c|c|c|c|c|}
\hline Strategie & Struktur & Kosten & Probleme & Bemerkungen \\
\hline Ein Zentralspital & $\begin{array}{l}\text { Case load; Schwerpunkt- } \\
\text { bildung; ein Chefarzt } \\
\text { pro Fach }\end{array}$ & $\begin{array}{l}\text { Hohe Investitionen } \\
\text { für Landerwerb und Bau; } \\
\text { anderweitige Verwendung } \\
\text { der Immobilien }\end{array}$ & Economy of scale; Politik & Modell Utopia \\
\hline $\begin{array}{l}\text { Zwei Akutspitäler } \\
\text { (standortübergreifende } \\
\text { integrierte Kliniken) }\end{array}$ & $\begin{array}{l}\text { Spitalübergreifende } \\
\text { Synergien; ein oder zwei } \\
\text { Chefärzte pro Fach }\end{array}$ & $\begin{array}{l}\text { Infrastruktur für Kommuni- } \\
\text { kation und Transport; } \\
\text { Komplexität des Betriebs }\end{array}$ & Kultur; Führung/Kontrolle & Modell Center of excellence \\
\hline $\begin{array}{l}\text { Zwei diversifizierte } \\
\text { Akutspitäler }\end{array}$ & $\begin{array}{l}\text { Patientennähe, Einzugs- } \\
\text { gebiet, Interdisziplinarität, } \\
\text { Flexibilität, Weiterent- } \\
\text { wicklung, Effizienz, zwei } \\
\text { Chefärzte pro Fach }\end{array}$ & $\begin{array}{l}\text { Doppelspurigkeiten } \\
\text { Auslastung }\end{array}$ & Vorhaltekosten; Case load & Modell Retail \\
\hline $\begin{array}{l}\text { Zwei Akutspitäler mit } \\
\text { Schwerpunktkliniken nur } \\
\text { an einem Standort }\end{array}$ & $\begin{array}{l}\text { Case load; Schwerpunkt- } \\
\text { bildung; ein Chefarzt } \\
\text { pro Fach }\end{array}$ & $\begin{array}{l}\text { Restrukturierung; } \\
\text { bauliche Massnahmen }\end{array}$ & $\begin{array}{l}\text { Patientenströme; } \\
\text { Interdisziplinarität }\end{array}$ & Modell Linkes Zürichseeufer \\
\hline
\end{tabular}


Das Gesundheitsamt ist mit Kontrollaufgaben betraut. Dabei müssen Investitionsprojekte von mehr als Fr. 500 000.- vom Verwaltungsrat dem DFS obligatorisch zur Stellungnahme vorgelegt werden. Die diesbezüglichen Entscheidungen des DFS sind auch für den Verwaltungsrat bindend. Diese Konstruktion relativiert den Effekt der Privatisierung, indem die Freiheit der Organe der Gesellschaft eingeschränkt wird.

\section{Finanzielles}

Die STGAG entwickelte sich finanziell ausgeglichen und damit erfreulich, insbesondere konnten die Folgen des TARMED ohne grössere Blessuren verkraftet werden.

\section{Auswirkungen auf die TVLS}

Die TVLS spielte im Umwandlungsprozess eine grosse Rolle als einzige Institution, in der die Ärzteschaft der teilweise konkurrenzierenden Institutionen (vor allem KSF und KSM) vertreten war. Der Präsident der TVLS sah die grösste Gefahr in einem Auseinanderdividieren der Ärzteschaft in den Fragen des Kaderarztvertrags, befürchtete unüberbrückbare Gegensätze und Feindschaften. Dies alles ist nicht eingetreten. Im Gegenteil nahmen Geschlossenheit und Corpsgeist innerhalb der TVLS in beträchtlichem Masse zu.

\section{Ausblick}

Die STGAG und ihre Kaderärzte haben den Übergang in eine Aktiengesellschaft erfolgreich vollzogen, die Kaderärzte konnten viele ihrer Postulate einbringen, sind in den Organen der Gesellschaft angemessen vertreten, sind als eigenständige Arbeitnehmerorganisation anerkannt, und die Strukturen haben sich konsolidiert. Um dieses Resultat zu erreichen, lag der Fokus im Organisatorischen und Strukturellen, wobei die medizinische Entwicklung zwangsläufig etwas hintangestellt wurde. Aktuell wird daran aber wieder gearbeitet, so dass der Zukunft durchaus mit einem gewissen Optimismus entgegengesehen werden kann.

\section{Lessons learned}

Die Kaderärzte eines privatisierten Spitalverbundes müssen sich in einer geeigneten Form, z. B. als Verein, organisieren, um Einfluss auf die Ausgestaltung neuer Organisationsformen nehmen zu können Die schon bestehende TVLS stellte im Falle der STGAG eine ideale Voraussetzung für die Handlungsfähigkeit der Ärzteschaft dar. Die TVLS ist an der Aufgabe in ihrem Zusammenhalt merkbar gestärkt worden.
Die Kaderärzte eines privatisierten Spitalverbundes sollen einen eigenständigen und von den Führungsorganen anerkannten Arbeitnehmerverband bilden

Dank der Anerkennung der TVLS als eigenständige Arbeitnehmervertretung konnte der speziellen Anstellungssituation der Kaderärzte Rechnung getragen werden, was zum Abschluss eines annehmbaren Kaderarztvertrags führte.

\section{Ärztliche Aspekte müssen im Verwaltungsrat vertreten sein}

Dieses Postulat wurde zunächst in der STGAG nicht verwirklicht, ist aber seit 2004 realisiert durch Einsitznahme eines unabhängigen auswärtigen Arztes in den Verwaltungsrat.

\section{Die Kaderärzte müssen in der Geschäfts-} leitung angemessen vertreten sein Bei der Gründung der STGAG war vorgesehen, dass zwei ärztliche Direktoren in die Geschäftsleitung eintreten. Alle vier ärztlichen Direktoren wurden dafür angefragt. Dabei verzichteten die beiden ärztlichen Direktoren des KSF und KSM aus eigenen Stücken auf die Einsitznahme, und zwar vor allem aus Befürchtungen, die auf sie zukommende Arbeitslast könnte ihre Funktionsfähigkeit als ärztliche Direktoren und Klinikchefs in Frage stellen. Gewählt wurden die ärztlichen Direktoren der PDT und TKK, die gleichzeitig auch als Spitaldirektoren ihrer Institution fungieren. Die Organe und die Ärzteschaft haben gelernt und kamen zum Schluss, dass die ärztliche Repräsentanz mit zwei Mitgliedern in der Geschäftsleitung zu klein war, was einvernehmlich korrigiert wurde.

Der Übergang in eine Aktiengesellschaft und die Führung derselben erfordern ein sehr grosses und uneingeschränktes Engagement der Ärzteschaft, insbesondere der Mitglieder der Geschäftsleitung sowie der Führungsorgane der Kaderarztorganisation (hier TVLS)

Nur bei angemessener Repräsentanz der Ärzteschaft in den Entscheidungsgremien wird die Betrachtungsweise der Führungsaufgaben auf medizinische statt nur administrative Belange und die damit verbundenen Strategien gelenkt. Das Mass des notwendigen Engagements und der Arbeitsaufwand für die davon betroffenen Kaderärzte wurde zu Beginn massiv unterschätzt. Eine Einsitznahme von Kaderärzten in die Geschäftsleitung führt zu einer Zerreissprobe der davon betroffenen Klinikleiter an der Trennlinie Aufgaben Geschäftsleitungsmitglied und Führung der eigenen Klinik. Die betroffenen Personen haben dafür interne Massnahmen ergriffen, 
wie z.B. die Einstellung eines Assistenten des ärztlichen Direktors, und zeigen sich der neuen Situation gewachsen. Zurückhaltung im Engagement ist absolut fehl am Platz.

Auch nach der Umwandlung in eine Aktiengesellschaft, die mehrheitlich oder vollständig in Besitz des Staates bleibt, ist der Handlungsspielraum der Organe der Gesellschaft eingeschränkt

Es ist eine Illusion zu glauben, ein privatisierter Spitalverbund werde völlig unabhängig von Staatsinterventionen. Vielmehr zeigt das Beispiel der STGAG, dass der Einfluss des Staates erheblich bleibt und dass damit die Beweglichkeit der Institution im geringeren Grade zunimmt als ursprünglich erhofft.

Bei der Umwandlung eines Spitalverbundes in eine Aktiengesellschaft entstehen zusätzliche Führungsebenen. Dies schafft einen zusätzlichen Kommunikations- und Informationsbedarf

Die Gründung der STGAG führte zu zwei zusätzlichen Führungsebenen gegenüber der früheren Struktur (Geschäftsleitung und Verwaltungsrat). Der damit verbundene Kommunikations- und Informationsbedarf wurde stark unterschätzt. Neben der Dienstwegkommunikation bedarf es zusätzlicher, ausserhalb des Dienstwegs liegender Kommunikations- und Informationskanäle, ohne die neue Schwerfälligkeit entsteht. Der damit verbundene Aufwand belastet die Kaderärzte zusätzlich. Die erhoffte Entlastung im Administrativbereich ist noch nicht realisiert, wird derzeit aber aktiv angegangen.

Der Beizug externer Berater kann entscheidende Impulse geben. Voraussetzung dafür ist ein unvoreingenommenes Herantreten der Berater an die gewachsenen Strukturen unter Berücksichtigung der Erfahrung der Kaderärzte. Eine Mischung von Analysekultur und Erfahrungskultur ist einer reinen Analysekultur vorzuziehen Die externen Berater der STGAG analysierten die STGAG vor allem unter den Gesichtspunkten, den Case load einzelner Kliniken zu vergrössern und Synergien zwischen den Spitälern zu schaffen. Die Erfahrung der Kaderärzte und deren Be- denken wurden zunächst in zu geringem Masse berücksichtigt, und es wurde vorwiegend an einem einzigen Strategiemodell gearbeitet, wobei andere denkbare Modelle weniger Berücksichtigung fanden. Die daraus entstehenden Meinungsverschiedenheiten führten zu Unstimmigkeiten und Zeitverlusten. Es wäre deshalb sinnvoll gewesen, frühzeitig mehrere mögliche Strategiemodelle ernsthaft zu prüfen.

Die Umwandlung eines Spitalverbundes in eine Aktiengesellschaft bindet erhebliche Managementkapazitäten. Bei der Auswahl von Projekten ist im Anfangsstadium eine Beschränkung auf eine kleine Anzahl mit hoher Realisierungschance sinnvoll In der STGAG konnte von den ursprünglich geplanten Projekten nur ein Teil realisiert werden. Es handelte sich dabei fast ausschliesslich um solche mit einer a priori hohen Wahrscheinlichkeit der Realisierung. Die Arbeit an den nicht realisierten Projekten band erhebliche Ressourcen des Managements und der Ärzteschaft.

\section{Fazit}

- Die STGAG ist beispielhaft für die Umwandlung eines öffentlichen Spitalverbundes in eine privatrechtliche Aktiengesellschaft. Die ins Auge gefassten Ziele wurden teilweise erreicht, wichtige, besonders medizinische Ziele müssen aber weiterbearbeitet werden.

- Die Kaderärzte der STGAG haben in massgeblicher Weise an der Umwandlung mitgearbeitet, diese teilweise erfolgreich mitgeprägt und konnten wichtige Postulate durchsetzen, u.a. einen annehmbaren neuen Kaderarztvertrag.

- Der Aufwand dafür war viel grösser als vermutet und bedingte den selbstlosen Einsatz einer grossen Zahl von Kaderärzten. In Zukunft wird dieser Einsatz nicht kleiner und wird sich zunehmend auf die Realisierung medizinischer Projekte und Fortschritte fokussieren.

- Die Thurgauische Vereinigung Leitender Spitalärzte geht gestärkt und geeint aus den vielfältigen Auseinandersetzungen mit der Umwandlung hervor, auch dies eine Voraussetzung für nachhaltigen Erfolg in der $\mathrm{Zu}$ kunft. Die Lessons learned ersetzen alte Paradigmen durch neue. 